\title{
EDITORIAL
}

\section{Metabolomics: the final frontier?}

\author{
Timothy D Veenstra*
}

The connection between genomics, proteomics and metabolomics is evident in even the most simplistic of scientific models. Genes give rise to mRNA. Proteins are translated from mRNA and then proceed to carry out a myriad of functions within the cell, including the metabolism of small molecules such as glucose and adenosine triphosphate. Not that many years ago, scientists used to study the 'big 4' biomolecules under the guise of genes, transcripts, protein and metabolites. The last decade of biomedical research, however, has been witness to the growth of the 'omics' industries. Genomics, transcriptomics and proteomics have become core technologies within almost every major academic or industrial research program around the world. What was missing was the final piece of the omics puzzle: metabolomics.

\section{Mass spectrometry or nuclear magnetic resonance?}

From a technology perspective, metabolomics has come along at precisely the right time. The two major technologies used to gather metabolomics data, mass spectrometry (MS) and nuclear magnetic resonance (NMR) spectroscopy, have both reached fantastic heights of data gathering capability $[1,2]$. Comparatively speaking, however, MS and NMR spectroscopy have their own specific advantages and disadvantages when conducting metabolomic studies. The main advantage of MS is sensitivity, as state-of-the-art mass spectrometers can detect analytes routinely in the femtomolar to attomolar range. Coupling MS with liquid chromatography (LC) or gas chromatography (GC) enables the measurement of hundreds of individual species within a single sample. The combination of mass accuracy and real-time tandem MS available with many mass spectrometers, along with increasingly comprehensive databases, is making the identification of these metabolites more routine. One of the major weaknesses of MS in metabolomics is quantification. The MS signal intensity of any compound is affected by the type of sample preparation used and its molecular

*Correspondence: veenstrat@mail.nih.gov

Laboratory of Proteomics and Analytical Technologies, SAIC-Frederick, Inc.,

Frederick National Laboratory and Research Center, Frederick, MD 21702, USA environment. Adding known amounts of internal isotopelabeled standards enables accurate quantification for specific molecules; however, this strategy is impractical for purely discovery-driven metabolomics research. Most studies rely on comparing peak area or intensity to locate differences in the relative abundance of specific metabolites between samples. However, these measurements can suffer from a lack of accuracy and precision.

The major weaknesses of MS are the major strengths of NMR spectroscopy. The peak area of a compound in the NMR spectrum is directly related to the concentration of specific nuclei (for example, ${ }^{1} \mathrm{H},{ }^{13} \mathrm{C}$ ), making quantification of compounds in a complex mixture very precise. A metabolite detected as being more abundant in a specific sample can be identified either through the resonance positions of its nuclei in the NMR spectrum, or through the application of various pulse-sequences such as total correlation spectroscopy, heteronuclear single quantum coherence and heteronuclear multiple bond correlation. Another underappreciated character of NMR spectroscopy is its versatility for analyzing metabolites in the liquid state (serum, urine and so on), in intact tissues (for example, tumors) or in vivo. Unfortunately, sensitivity, which is the major strength of MS, is the major weakness of NMR spectroscopy. Although cryogenically cooled probe technology, higher field-strength superconducting magnets [3] and miniaturized radiofrequency coils [4] have increased sensitivity, NMR spectroscopy is still orders of magnitude less sensitive than MS.

While metabolomics is less mature than genomics and proteomics, it is already making a major impact in a wide variety of scientific areas, including newborn screening, toxicology, drug discovery, food safety and biomarker discovery (Figure 1). As with genomics and proteomics, most of the pressure will be on metabolomics to find biomarkers of diseases such as cancer. Investigators have already shown the potential promise of metabolomics in this area. For example, Sreekumar et al. [5] used LC-MS and GC-MS to profile 42 tissue, 110 urine and 110 plasma samples from patients affected with benign prostate disease, clinically localized prostate disease and metastatic disease. Not only were they able to distinguish these three conditions based on the NMR data, but they found that a specific metabolite, sarcosine, was highly 


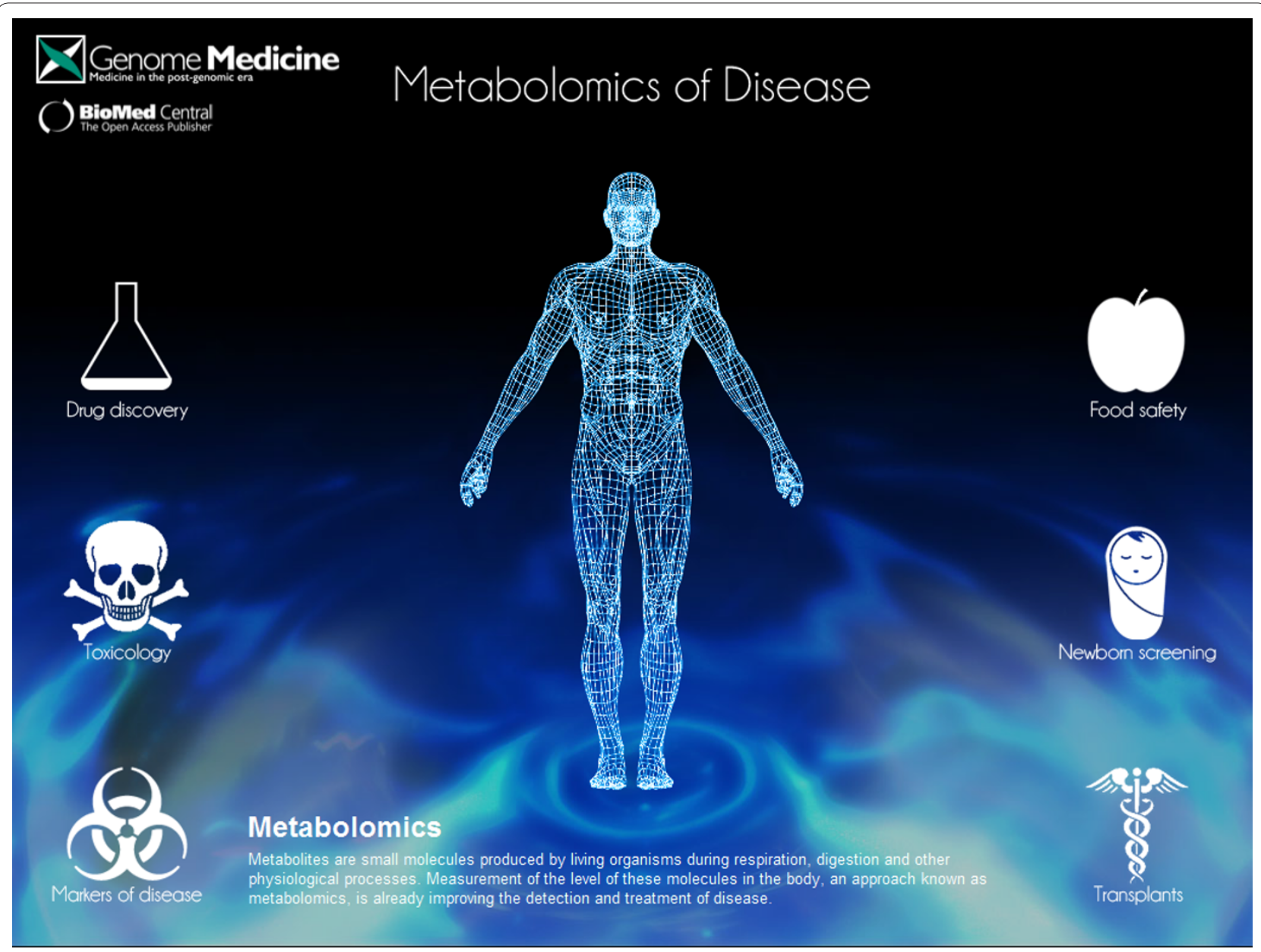

Figure 1. Metabolomics of disease. Metabolomics technologies are making a major impact on a wide variety of scientific and clinical areas, including newborn screening, toxicology, drug discovery, food safety and biomarker discovery. Please use the following link to access the interactive version of this graphic [7].

increased in concentration during prostate cancer progression to metastasis. This increased sarcosine level could be detected non-invasively in urine. In an ovarian cancer study, Dr Olivier Fiehn's laboratory conducted a metabolomics screening of 66 invasive ovarian carcinoma tissues and 9 borderline tumors of the ovary [6]. Over 50 identified metabolites were shown to differ significantly between the sample cohorts. Many of these metabolites play a role in purine and pyrimidine metabolism, glycerolipid metabolism and energy metabolism. The next challenge with this study, as with every biomarker discovery study using omics technology, are to conduct validation studies to determine if any of the metabolites can be used to reliably diagnose the disease.

\section{The future of metabolomics}

Metabolomics has a unique opportunity to impact discovery-driven science in a way that genomics, transcriptomics and proteomics could not fully exploit.
Metabolomics is maturing at a time when the other three omic technologies are much further advanced, and therefore we now have a greater opportunity to integrate metabolomics data with those obtained for the other 'big 4' biomolecules. If we go back to our simplistic model of gene $\rightarrow$ transcript $\rightarrow$ protein $\rightarrow$ metabolite, our greatest chance in finding truly useful disease biomarkers will be studies that show correlation between biomolecules at all four levels. With genomic, transcriptomic and proteomic data collection and analysis further advanced, it seems logical for any metabolomics discovery study to add results from those whenever possible. Years of effort have demonstrated, particularly in the fields of transcriptomics and proteomics, that working in technological 'silos' is an inefficient way to solve challenging biological problems.

A goal of the omic technologies is to be able to apply them in a clinical setting to evaluate or monitor the health status of patients. Of the two major technologies utilized in metabolomics, MS is more likely than NMR 
spectroscopy to play a greater role in the clinic. MS is already being applied routinely in clinical and diagnostic laboratories. In its present role, the ability of MS to directly measure a specific metabolite(s) in a biological sample is employed using methods primarily built upon isotope-dilution-MS. While most clinical MS assays are conducted in reference laboratories, this scenario is likely to change over the next few years as mass spectrometers became a standard laboratory instrument and the number of scientists familiar with this technology continues to increase. While magnetic resonance imaging (MRI) is widely used within hospitals, it is not the same technology as the NMR spectroscopy described in this editorial.

The ability to translate discovery into assays that can be applied routinely within the clinic will have an enormous impact on public health. In particular, the cry for diseasespecific biomarkers continues to be heard, as healthcare professionals have been promised for over a decade that omic technologies would meet this need. Can metabolomics be the science that finally conquers the biomarker discovery challenge? On its own, it is likely to have no more success than proteomics. On its own, it will flood the literature with hundreds of studies that publish hundreds of biomarkers that show potential but never graduate to validation studies. However, integrating data from genomic and/or proteomic studies that corroborate metabolomics findings will provide the evidence needed to confidently recognize those biomarkers that are worth the resources required for validation. The hope is that metabolomics can learn from the other omic techniques and not repeat many of the same mistakes that have been made in which a lot of data were collected but little information was gleaned.

\section{Abbreviations}

GC, gas chromatography; LC, liquid chromatography; MS, mass spectrometry; NMR, nuclear magnetic resonance.
Competing interests

The author declares that he has no competing interests.

\section{Acknowledgements}

This project has been funded in whole or in part with federal funds from the National Cancer Institute, National Institutes of Health, under contract N01-CO-12400. The content of this publication does not necessarily reflect the views or policies of the Department of Health and Human Services, nor does mention of trade names, commercial products or organizations imply endorsement by the US Government. The figure was designed by Warwick Bromley and the text for the figure and online graphic was provided by Maria Hodges.

Published: 30 April 2012

\section{References}

1. Sands CJ, Coen M, Ebbels TM, Holmes E, Lindon JC, Nicholson JK: Datadriven approach for metabolite relationship recovery in biological ${ }^{1} \mathrm{H}$ NMR data sets using iterative statistical total correlation spectroscopy. Anal Chem 2011, 83:2075-2082.

2. Patti GJ, Yanes O, Siuzdak G: Innovation: Metabolomics: the apogee of the omics trilogy. Nat Rev Mol Cell Biol 2012, 13:263-269.

3. Felli IC, Brutscher B: Recent advances in solution NMR: fast methods and heteronuclear direct detection. Chemphyschem 2009, 10:1356-1368.

4. Kentgens AP, Bart J, van Bentum PJ, Brinkmann A, van Eck ER, Gardeniers JG, Janssen JW, Knijn P, Vasa S, Verkuijlen MH: High-resolution liquid- and solidstate nuclear magnetic resonance of nanoliter sample volumes using microcoil detectors. J Chem Phys 2008, 128:052202.

5. Sreekumar A, Poisson LM, Rajendiran TM, Khan AP, Cao Q, Yu J, Laxman B, Mehra R, Lonigro RJ, Li Y, Nyati MK, Ahsan A, Kalyana-Sundaram S, Han B, Cao X, Byun J, Omenn GS, Ghosh D, Pennathur S, Alexander DC, Berger A, Shuster JR, Wei JT, Varambally S, Beecher C, Chinnaiyan AM: Metabolomic profiles delineate potential role for sarcosine in prostate cancer progression. Nature 2009, 457:910-914

6. Denkert C, Budczies J, Kind T, Weichert W, Tablack P, Sehouli J, Niesporek S, Könsgen D, Dietel M, Fiehn O: Mass spectrometry-based metabolic profiling reveals different metabolite patterns in invasive ovarian carcinomas and ovarian borderline tumors. Cancer Res 2006, 66:10795-10804

7. Metabolomics of disease [http://www.biomedcentral.com/sites/3008/ Metabolomics/Metabolomics.html]

doi:10.1186/gm339

Cite this article as: Veenstra TD: Metabolomics: the final frontier? Genome Medicine 2012, 4:40. 